SMAD, Rev. Eletrônica Saúde Mental Álcool Drog.

\title{
Política de redução de danos e o cuidado à pessoa em situação de rua
}

Lorenna Saraiva Viana ${ }^{1}$

(D) https://orcid.org/0000-0003-1496-5164

Eliany Nazaré Oliveira ${ }^{2}$

(D) https://orcid.org/0000-0002-6408-7243

Maria Suely Alves Costa ${ }^{1}$

(D) https://orcid.org/0000-0002-3545-0613

Claudine Carneiro Aguiar ${ }^{3}$

(D) https://orcid.org/0000-0001-7679-1564

Roberta Magda Martins Moreira ${ }^{1,4}$

(D) https://orcid.org/0000-0002-8225-7576

Andriny Albuquerque Cunha ${ }^{1}$

(D) https://orcid.org/0000-0003-4132-1225

1 Universidade Federal do Ceará, Sobral, CE, Brasil.

2 Universidade Estadual Vale do Acaraú, Sobral, CE, Brasil.

3 Prefeitura Municipal de Sobral, Sobral, CE, Brasil.

${ }^{4}$ Bolsista da Coordenação de Aperfeiçoamento de Pessoal de Nível Superior (CAPES), Brasil.
Objetivo: descrever as oficinas realizadas com os profissionais envolvidos no cuidado à pessoa em situação de rua sobre a Política de Redução de Danos, tendo como referencial o Arco de Maguerez. Método: pesquisa intervenção desenvolvida com doze profissionais do Centro de Referência Especializada à pessoa em situação de rua e do Centro de Atenção Psicossocial Álcool e outras Drogas, tendo como referencial o Arco de Maguerez. Resultados: surgiram desafios relacionados ao estigma; fortalecimento da autonomia e fragilidades na articulação intersetorial. Citou-se também a parceria com o serviço especializado, Residência e Liga Interdisciplinar em Saúde Mental. Realizaram-se também momentos teórico-reflexivos e construção de um fluxo de cuidado. Conclusão: compreendese a importância de atividades de consciência crítica com os profissionais no intuito de aperfeiçoar o cuidado no âmbito da Redução de Danos.

Descritores: Educação; Redução de Danos; Cuidado; Pessoas em Situação de Rua.

\section{Como citar este artigo}

Viana LS, Oliveira EN, Costa MSA, Aguiar CC, Moreira RMM, Cunha AA. Harm reduction policy and caring for the homeless. SMAD, Rev Eletrônica Saúde Mental Álcool Drog. 2020;16(2):1-9. doi:https://dx.doi.org/10.11606/issn.1806-6976.smad.2020.157479 


\section{Harm reduction policy and caring for the homeless}

Objective: describe about workshops held with professionals involved in the care of the homeless on the Harm Reduction Policy, having as reference, the Arco de Maguerez. Method: intervention research developed with 12 professionals of homeless person Specialized Reference Center and Psychosocial Care Center Alcohol and other Drugs, having Maguerez Arc as reference. Results: challenges related with stigma appeared; autonomy strengthening and intersectoral articulation fragility. We also mentioned the partnership with specialized service, Mental Health Interdisciplinary League and Residency. We also promoted theoretical-reflexive moments and a care flux construction. Conclusion: we understand the importance of critical conscience activities with professionals aiming to improve care related to Harm Reduction.

Descriptors: Education; Harm Reduction; Care; Homeless People.

\section{Política de reducción de daños y cuidado de las personas sin hogar}

Objetivo: describir sobre los talleres realizados con profesionales involucrados en el cuidado de las personas en situación de calle sobre la política de reducción de daños, teniendo como referencia el arco de 0maguerez. Método: investigación de intervención desarrollada con doce profesionales del centro de referencia especializada a personas en situación de calle y del centro de atención psicosocial alcohol y otras drogas, teniendo como referencia el arco de maguerez. Resultados: surgieron desafíos relacionados al estigma; fortalecimiento de la autonomía y fragilidades en la articulación intersectorial. fue citada también la colaboración con el servicio especializado, residencia y liga interdisciplinar en salud mental. se realizaron también momentos teóricoreflexivos y la construcción de un flujo de cuidado. Conclusión: se comprende la importancia de actividades de consciencia crítica con los profesionales con el objetivo de perfeccionar el cuidado en el ámbito de la reducción de daños.

Descriptores: Educación; Reducción de Daños; Cuidado; Personas en Situación de Calle. 


\section{Introdução}

No contexto do uso de Substâncias Psicoativas (SPA) inserem-se alguns grupos específicos como as Pessoas em Situação de Rua (PSR), caracterizadas como uma população heterogênea composta por sujeitos com diferentes realidades, mas que têm em comum a condição de pobreza e a falta de pertencimento à vida. $\mathrm{O}$ uso de SPA surge como uma ferramenta para "amenizar" as consequências de um sintoma da insanidade social que exclui os cidadãos de seus direitos civis, políticos, sociais e econômicos ${ }^{(1)}$.

Nesse âmbito, essa prática se encontra imersa em diversos obstáculos que desafiam os princípios de universalidade, equidade e integralidade do Sistema Único de Saúde (SUS). Nesse sentido, muitos se encontram à margem das Redes de Atenção à Saúde (RAS) e à proteção social, sendo alvo de políticas assistencialistas, compensatórias e focalizadoras ${ }^{(2)}$.

Sendo assim, motivados pela cultura do preconceito e da exclusão de pessoas nessa situação, pode-se observar as seguintes barreiras: a burocratização do serviço e o preconceito por parte de alguns profissionais motivados pela aparência dos usuários (falta de roupas e higiene adequada), a ausência de documentos de identificação e endereço fixo, e/ou pela frequente condição de uso abusivo ou excessivo de álcool, crack e outras drogas (2). Dessa maneira, percebe-se que o estigma, enquanto estereótipo segregador, construído em torno de pessoas que vivem nas ruas que fazem uso de SPA, resulta no julgamento moral da conduta dos sujeitos a partir desses pontos ${ }^{(3)}$.

Para tanto, têm-se a Política de Redução de Danos (PRD), na perspectiva do modelo psicossocial, considerada o principal pilar da atenção aos usuários de SPA. Caracteriza-se como conjunto de ações de saúde pública voltadas para minimizar as consequências adversas causadas pelo uso abusivo dos psicoativos. Pauta-se na busca pela melhoria da qualidade de vida dos sujeitos, com o fortalecimento da autonomia, noções de cuidado e de autocuidado contextualizadas e compartilhadas ${ }^{(4)}$.

Nesse cenário, o objetivo do estudo foi descrever as oficinas realizadas com os profissionais envolvidos no cuidado à PSR sobre a PRD, tendo como referencial o Arco de Maguerez.

\section{Método}

O estudo, de abordagem qualitativa, foi construído a partir dos fundamentos teóricos da PesquisaIntervenção, que é caracterizada como uma tendência de pesquisa participativa, onde se busca investigar a vida das coletividades dentro da sua diversidade qualitativa, com o intuito de desenvolver propostas, diante das experiências sociais, de intervenções micropolítica(5).

As intervenções ocorreram de agosto a dezembro de 2018, no Centro de Referência à Pessoa em Situação de Rua (CENTRO POP) e no Centro de Atenção Psicossocial Álcool e outras Drogas (CAPS AD) do município de Sobral-Ceará. Ressalta-se que os dois dispositivos desenvolvem suas atividades em espaços diferentes.

O estudo envolveu, ao total, doze participantes, o que corresponde a cerca de $80 \%$ dos profissionais de cada serviço, sendo seis do CENTRO POP, incluindo-se um advogado, dois psicólogos, três assistentes sociais e seis do CAPS-AD (uma enfermeira, duas assistentes sociais, duas psicólogas e um profissional de educação física).

Foram desenvolvidas seis oficinas (três em cada serviço), realizadas em momentos diferentes, de acordo com a organização e disponibilidade dos dispositivos. Sendo assim, as intervenções com os profissionais do CENTRO POP ocorreram ao final do expediente e as oficinas com os profissionais do CAPS AD aconteceram ao longo das rodas setoriais do serviço, reuniões destinadas para organização do processo de trabalho, discussões dos casos mais complexos e para momentos de Educação Permanente em Saúde (EPS).

Utilizaram-se, como critérios de inclusão, ser profissionais com ensino superior completo que estivessem atuando nos dispositivos de cuidado há pelo menos um ano e que aceitassem participar, voluntariamente, da intervenção. Os critérios de exclusão incluíam anão possuir ensino superior completo, não estar nos serviços há pelo menos um ano, estar de licença (premia, maternidade ou atestado médico), estar de férias, bem como haver escolhido não participar da intervenção.

O planejamento e a implementação das intervenções foram fundamentadas na Metodologia da Problematização, tendo como referencial teórico o Arco de Maguerez, que propõe uma abordagem da realidade por meio de cinco etapas: observação da realidade; pontos-chave; teorização; hipóteses de solução e aplicação à realidade ${ }^{(6)}$.

A Metodologia da Problematização é configurada como uma estratégia de ensino que estimula o pensamento crítico e reflexivo, articulando a teoria com a prática. Portanto, a busca pela tomada de decisão está focada na discussão em grupo e na reflexão de problemas (de acordo com a realidade do contexto em que os participantes estão inseridos)(7).

Para melhor sistematização das informações, o trabalho foi organizado em três etapas: observação e problematização da realidade encontrada no processo de cuidado à PSR (primeira oficina de cada serviço), oficina teórico/prático sobre redução de danos (segunda oficina de cada serviço) e reflexões sobre 
a articulação do cuidado por meio do Mapa Falante (terceira oficina de cada serviço).

O estudo obedeceu aos princípios da resolução do Conselho Nacional de Saúde (CNS) 466/12, sobre pesquisa envolvendo seres humanos, (autonomia, beneficência, não maleficência e justiça) ${ }^{(8)}$. Constitui-se como um recorte de uma pesquisa de término de conclusão de uma Residência Multiprofissional em Saúde Mental e obteve aprovação do Comitê de Ética e Pesquisa da Universidade Estadual Vale do Acaraú - UVA, sob o parecer de $n^{\circ}$ 3.002.885.

Ademais, para que os nomes dos participantes fossem resguardados, os profissionais foram identificados por meio de um sentimento associado ao dispositivo em que trabalham.

\section{Resultados e Discussão}

Para iniciar a aplicação do Arco de Maguerez, ocorreu a observação da realidade no processo de cuidado à pessoa em situação de rua, que consistiu na participação ativa dos sujeitos, para um olhar atento e sensível à realidade, efetuando, assim, uma primeira leitura da qual o tema estava inserido. Portanto, em cada uma das primeiras oficinas de cada serviço, foi realizado um momento vivencial com os profissionais, a fim de se visualizar os diversos contextos em que vivem as PSR.

Após as reflexões iniciais, deu-se início à segunda etapa do Arco de Maguerez na primeira oficina, onde se tentou identificar os pontos-chave acerca da realidade de trabalho dos profissionais dos serviços. Utilizaram-se as seguintes perguntas norteadoras: Quais os principais desafios encontrados? Quais os principais fatores que podem contribuir nesse cuidado? Como se dá o fluxo do cuidado no âmbito da política de atenção integral em álcool e outras drogas?

Diante disso, identificaram-se alguns pontos fundamentais para serem trabalhados nas oficinas posteriores. A partir das falas, pontuaram-se as seguintes dificuldades no cuidado à PSR: particularidades do público; estigma e preconceito e o fortalecimento da autonomia dos usuários.

Acerca das problemáticas do cuidado, a maior parte dos profissionais apontou que o desenvolvimento do trabalho junto à PSR é desafiador devido às características e particularidades dessa população, tais como o nomadismo. Este pode ser compreendido como situação permanente de circulação itinerante da PSR, por diferentes regiões, que dificulta a continuidade do

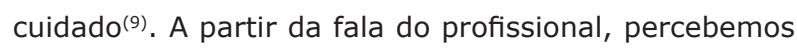
essa questão: Adequar estratégias de cuidado ao modo de vida deles é um pouco complicado, é difícil porque eles estão em situação de rua. O estar em situação de rua é um desafio por si só. (Mansidão POP).
Ainda relacionado ao nomadismo, considerado como uma particularidade da PSR ficou evidenciada nas falas, principalmente entre os profissionais do CAPS AD, a ausência de uma referência. Neste caso, a referência está relacionada à presença de uma moradia fixa, de um familiar e/ou algum responsável pelo acompanhamento do cuidado no domicílio(10), como foi constatado na seguinte frase: o principal foco daqui é o familiar, é buscar o familiar para poder estar dando apoio a essa pessoa, para poder estar ajudando no cuidado (Alegria AD).

Outro aspecto retratado, por todos os profissionais, principalmente os do CENTRO POP, diz respeito ao preconceito e ao estigma, muito presente em relação à PSR, dificultando a garantia de direitos em diversas perspectivas. A gente vê também a questão do preconceito que a gente sabe que existe, um preconceito e um estigma muito grande (Parceria POP).

Sobre essa problemática, aponta-se que tanto a pessoa estigmatizada como que a prática pode desenvolver mecanismos de proteção que se manifestam mediante condutas ou comportamentos de afastamentos ${ }^{(11)}$. Diante disso, identifica-se que a PSR está vulnerável ao preconceito pela sua condição social, ou seja, a partir da sua maneira de viver, como relacionada ao fato de adquirirem determinadas doenças próprias dessa condição de vida. Sendo assim, muitas PSR não conseguem, muitas vezes, ter acesso a uma rede de suporte de saúde, social, emocional, afetivo nem prático ${ }^{(10)}$.

Essa perspectiva foi prevalente entre os profissionais do CENTRO POP diante da dificuldade que eles visualizam das pessoas que são acompanhadas pelo serviço quando necessitam de algum atendimento no setor da saúde, atrelada principalmente ao estigma e preconceito a esse público. Eles acham que se forem em um posto de saúde, se não for acompanhado pela equipe, o pessoal vai tratar eles mal (Maturidade POP).

Diante disso, faz-se necessário efetivar as políticas públicas de acesso à saúde para a população, em especial a PSR. Assim, é preciso valorizar as particularidades e subjetividade de cada indivíduo e compreendê-los enquanto cidadãos, que possuem os mesmos direitos de cidadania; promover questionamentos acerca dos problemas mais prevalentes, mostrando as dificuldades nas inter-relações, entre os processos de cuidado e modos de vida na rua; ter competência técnica e humana além de se ter sensibilidade por parte dos profissionais que atuam junto a esse público(12).

Apesar dessa dificuldade de acesso, aos demais serviços, os profissionais entrevistados procuram trabalhar a autonomia dos usuários dentro da perspectiva de uma possível resolução dos problemas mencionados e tentando minimizar suas demandas, conforme foi mencionado por um dos entrevistados: Às vezes tem a 
"tutelagem" que eles querem que a gente faça pra ir pra consulta, principalmente pra os usuários mais antigos, para levar até o CAPS ou levar em algum lugar. A gente tenta explicar, a gente aqui ta com a intenção de conseguir essa autonomia. Então, aqui, na assistência social tem sempre essa predominância e esse rigor de se criar autonomia (Felicidade POP).

A partir das discussões, nessa segunda etapa, identificam-se também algumas potencialidades referentes ao cuidado à PSR, tanto em aspectos gerais como referentes aos usuários, que fazem uso de alguma substância psicoativa. Foi apontada, por todos os serviços, a importância do próprio espaço físico e das atividades internas que acontecem em cada um dos locais, que articulam o cuidado; a parceria com a Residência Multiprofissional em Saúde Mental (RMSM) e a Liga Interdisciplinar em Saúde Mental (LISAM) que apoiam e reforçam o cuidado especializado a essa população.

No que concerne às atividades internas, que são realizadas nos dois serviços no âmbito da PSR, aponta-se o seguinte relato: Observamos o serviço em si mesmo para eles, a segurança, de higiene que a gente oferece pra eles, esse apoio que a gente tem em relação ao encaminhamento deles na saúde (Felicidade POP).

Em relação ao CAPS $A D$, foi discorrido, acerca do apoio do serviço, por meio da equipe multiprofissional, a intersetorialidade e suporte dos grupos no cuidado à PSR. Temos o suporte da equipe multi; o apoio intersetorial, a questão dos grupos, nós temos muita diversidade em relação aos grupos (Responsabilidade AD).

Nesse âmbito, reflete-se acerca da importância do CAPS AD, que, de acordo com a nova política nacional de álcool e outras drogas deve, preferencialmente, ser inspirada na abordagem direcionada à redução de danos. Nesse caso, o sujeito, antes passivo, passa a ser protagonista da sua condição de vida e de cuidado com uma política antagônica ao modelo de abstinência (alta exigência) de hospitais psiquiátricos e comunidades terapêuticas. Para tanto, deve-se trabalhar na lógica da intersetorialidade, multidisciplinaridade, acompanhamento em grupos, perspectiva territorial, intervenções e abordagens de questões relacionadas ao estigma(13).

Além disso, faz-se necessária a obtenção de informações sobre a clientela que demanda atendimento nos CAPS AD. Seguindo a lógica de cuidado singularizado e na busca de um melhor entendimento acerca do papel que o dispositivo ocupa junto à população e à rede de atenção(14).

Na maioria dos relatos, percebeu-se a importância da atuação da RMSM e da LISAM, nos dois serviços, mediante realização de atividades sistemáticas, que envolvem diversas perspectivas de cuidado à pessoa marginalizadas no âmbito da política de atenção integral em álcool e outras drogas. A gente ta com o grupo de Redução de Danos lá no CENTRO POP e a gente também consegue trazer os casos por meio da residência (Leveza AD).

Ainda na primeira oficina de cada dispositivo, no que concerne aos fluxos de cuidado à PSR, pode-se identificar que o modo de organização do cuidado está inter-relacionado aos desafios encontrados. A princípio, discutiu-se acerca do processo de trabalho e função de cada dispositivo em relação à PSR. Essa queixa foi colocada, principalmente, pelos profissionais do CENTRO POP, conforme pode ser observado na fala a seguir: A gente queria que os equipamentos entendessem qual o trabalho do CENTRO POP, a gente não é caridade, a gente trabalha em cima de políticas (Cumplicidade POP).

Discute-se que os serviços de saúde e assistência social reforçam, em seus discursos, limites de atuação, repercutindo em forte setorialização e hierarquização configurando um trabalho compartimentado. Sendo assim, as equipes atuantes nesses serviços, possuem diferentes entendimentos acerca da relação do processo de trabalho de ambos. Promovendo possíveis dúvidas e discordâncias entre a concepção de atuação e as atribuições de cada setor ${ }^{(15)}$.

Podem-se identificar muitas dificuldades dos profissionais do CAPS AD em estar organizando o cuidado nesse dispositivo. Primeiramente, percebe-se que não há uma linha de cuidado específica para a PSR, sendo assim, tende-se a diversas divergências dentro da equipe do serviço. Não tem nenhuma linha de cuidado específico não, foi até pensado uma época quando o médico daqui estava fazendo matriciamento por lá de alguma coisa para facilitar o fluxo deles aqui, alguma coisa assim (Leveza AD).

Essas dificuldades, por sua vez, vão ao encontro de algumas especificidades da PSR, apontadas pelos profissionais e que se constituem como obstáculos para a continuidade do acompanhamento no dispositivo. Eles têm um modo de funcionamento diferente, não são institucionalizados como a gente, a gente tem um canto fixo, um horário de abrir e fechar e eles são mais dinâmicos (Pureza AD).

Diante do exposto, têm-se a reflexão de que é necessário compreender as especificidades do trabalho com a PSR, com adaptação interna dos serviços em seus funcionamentos a fim de se responder às demandas desse grupo. Destaca-se a necessidade da construção de uma linha de cuidados para a PSR que seja pautada nas suas características, nos determinantes do processo saúde-doença, problemas clínicos e dificuldades enfrentadas na rede de cuidados intersetoriais(15).

Ao longo da oficina, pode-se observar também outro entrave no fluxo, relacionado à articulação dos serviços da assistência e saúde com as Comunidades Terapêuticas (CT). As falas dos profissionais do CENTRO POP retratam que o interesse no encaminhamento desses espaços, ainda, é muito presente entre as pessoas em situação de rua. Pode-se perceber que as 
CT surgem como estratégia de fuga de determinadas situações e não como uma estratégia intencionada aos amparos, de alguma forma, como mencionado na fala a seguir: Muitos chegam aqui na maior euforia que quer ir pra casa de recuperação que quando a gente vai ver por trás ta fugindo de alguma coisa, de algum ato que cometeu na rua, que só quer ir pra lá pra se esconder. Ele não quer realmente um tratamento (Parceria POP).

Um dos profissionais do CAPS AD apontou que muitos usuários chegam ao serviço, unicamente, para serem encaminhados às $\mathrm{CT}$. A maioria dos usuários que vêm ao acolhimento já chega tencionando pra irem pras comunidades terapêuticas e isso é muito forte. E eles falam que acha ruim porque demora o acesso, que precisa vir pros atendimentos (Responsabilidade AD).

$\mathrm{Na}$ terceira etapa do Arco é realizada a fundamentação teórica acerca da temática trabalhada(6). Sendo assim, na segunda oficina de cada serviço foi realizada uma linha temporal com os principais marcos teóricos acerca da PRD. Pode-se compreender que o conceito de redução de danos ainda é muito limitado e podendo ser mal interpretado por alguns profissionais. Na minha visão, essa palavra redução de danos foi mal trabalhada, mal construída, mal repassada, porque todos os dispositivos que foram falados, eles fazem algum tipo de redução de danos (Bondade AD).

Outra profissional discursou, acerca das questões sociais, religiosas e familiares que está envolvida em torno da PRD e que dificulta no fortalecimento dessa política nos serviços. E também é uma lógica construída né, perpassa as questões religiosas, as familiares, a redução de danos é uma política difícil de compreender, partindo de uma sociedade que está atravessada por diversas questões (Leveza AD).

Nesse sentido, debate-se que os dispositivos de poder (relacionados à justiça e ao exercício da lei) assumem uma postura criminalizadora e penalizadora em relação ao usuário de SPA(16).

Também apontaram limitações que alguns profissionais possuem dentro do contexto de alguns aspectos da PRD, como a autonomia do sujeito em decidir a melhor forma de seguir de tratamento, seja a partir da abstinência, da redução do consumo ou de qualquer outra estratégia. Eu acho que às vezes não é nem má vontade do profissional em entender nwé, mas é da própria compreensão de estar na lógica da abstinência, de que o ideal pro paciente é ficar abstinente, então, se a gente parte dessa lógica, fica muito difícil trabalhar redução de danos e principalmente pensar que o melhor pra gente não é o melhor pra ele (Leveza AD).

Compreende-se a importância de que se reflita sobre o fato de que não há um tratamento único e que seja ideal para todas as pessoas; que se considere às necessidades dos indivíduos, contemplando, não somente à questão do uso de SPA, mas a perspectiva integral e da diversidade contextual, além da presença de um cuidado multiprofissional, que deve ser avaliado com frequência e que preserve a autonomia e o desejo desse sujeito ${ }^{(17)}$.

Associado a linha do tempo, foi realizada uma oficina prática com os profissionais dos dois serviços, com o objetivo de discutir algumas estratégias possíveis que possam ser utilizadas para promover a melhoria na qualidade de vida dos usuários acompanhados pelos serviços. Ademais, buscou-se trazer uma perspectiva mais ampla, direcionada também para a importância de garantir a autonomia e a cidadania desses sujeitos, em relação ao uso e a forma de relacionamento destes com as substâncias.

Observa-se, o fortalecimento da PRD junto aos usuários. No entanto, há alguns desafios a serem superados, tais como a falta de financiamento para disponibilização de insumos aos usuários. A partir da fala abaixo, identifica-se um exemplo de profissional que compra insumo, por conta própria, para dar continuidade a esse tipo de cuidado no serviço: Ultimamente eu tenho comprado o papel seda, está lá na sala da coordenação e os usuários que fazem o uso de fumo, geralmente eu dou (Gratidão $A D)$.

Ao longo dessa oficina, diante de um diálogo aberto com os profissionais, pode-se compreender que dentro desse complexo cuidado, pautado na PRD à pessoa em situação de rua, existem diversos aspectos que precisam estar presentes, como: a construção de vínculos de confiança diante de uma prática de cuidado centralizado na escuta e no conhecimento do outro; o trato humanizado; reconhecimento de suas condições materiais e simbólicas de sua existência; desenvolvimento de um olhar que consiga se afastar de uma perspectiva normativa e moralizante. A gente tenta trabalhar com eles e pra eles. É por meio de um trabalho mútuo, uma troca, dando que se recebe. O jeito que eu falo com eles, depende de tudo. Precisa ser uma troca de cada um de nós com o usuário (Felicidade POP).

Nesse sentido, torna-se fundamental a concretização das tecnologias leves, pautadas nas relações humanas, como a produção de vínculo, autonomização, acolhimento, no encontro entre o profissional e o usuário. A partir destas tecnologias, pautadas no diálogo aberto e respeito às singularidades que se constrói e se consolida a positividade entre os sujeitos envolvidos nas relações do cuidado(18). Reflete-se, portanto, a importância dos profissionais estarem disponíveis, de se ter sensibilidade para se tratar e cuidar de pessoas buscando seus aspectos subjetivos, histórias e necessidades de vida das PSR.

$\mathrm{Na}$ terceira oficina de cada serviço foi utilizada a Metodologia do Mapa Falante como estratégia para o levantamento de hipótese de soluções (quarta etapa do Arco de Maguerez). Nesse sentido, o mapa falante vem a 
se caracterizar como potente instrumento para fazer uma leitura da realidade a partir de suas múltiplas dimensões. Caracteriza-se como expressão de um território, que considera a representação objetiva e simbólica do espaço vivo, permitindo a visualização de informações de uma localidade e a representação de áreas de relevância e interesse para um conjunto de atores ${ }^{(19)}$.

Em primeiro momento, foi apresentado um caso hipotético de uma usuária em situação de rua que havia demandas clínicas e sociais relacionadas ao uso de substâncias. Diante disso, os participantes, construíram um possível fluxo de cuidado a esse usuário, tendo em vista a articulação por meio dos dispositivos da Rede de Atenção à Saúde (RAS), equipamentos da assistência social assim como os mecanismos informais de cuidado.

Os profissionais, dos dois serviços, apontaram a importância da articulação junto à Atenção Primária à Saúde (APS), como principal porta de entrada para o cuidado em saúde e reordenação dos outros processos, em parceria com a equipe especializada e a assistência. Acredito que a Atenção Básica precisa estar bem na frente do mapa (Felicidade POP).

Nessa perspectiva, a partir da lógica da APS, entende-se que a essência desse ponto de atenção à saúde deve estar pautada no acolhimento e responsabilização pelos problemas de saúde dos usuários. Utilizando-se de relações de vínculo e capacidade de escuta para se garantir a continuidade e longitudinalidade do atendimento à população adstrita. Além de constituir o território, como base para organização das práticas de cuidado, de acordo com as singularidades dos sujeitos ${ }^{(20)}$.

Ainda foi retratado, acerca da Unidade de Acolhimento (UA), que estava no mapa falante, mas que ainda não está em funcionamento no município, conforme apontado na frase a seguir: Então, o CENTRO $P O P$, que poderia ser um parceiro, tem o CREAS que poderia ser um parceiro, mas ai vai depender muito da sensibilização de cada um para ganhar forças para abrir a unidade de acolhimento, porque fica só o CAPS AD de ficar tencionando sobre a abertura, sobre fortalecer a política de redução de danos (Bondade AD).

Nessa fala, percebe-se a inquietação de um profissional ao retratar a necessidade dos serviços da assistência social, envolvidos no cuidado, estejam integrados em ganhar força a fim de tornar possível à abertura da UA (como local para acolhimento de usuários que estão em situações de grandes vulnerabilidades, seja sociais, familiares ou físicas) ${ }^{(21)}$.

Tensionou-se, como quarta e quinta fase do Arco, hipóteses de solução e aplicação à realidade, para que as discussões propostas nas intervenções fossem aplicadas à realidade, a fim de se buscar a melhoria e o aperfeiçoamento do cuidado desse público, na perspectiva da PDR.

\section{Conclusão}

Por meio da realização dessa pesquisa, percebeu-se que os dois dispositivos atuam com demandas relacionadas à PSR, mas mesmo atuando nesse grupo, identifica-se que as abordagens da saúde e da assistência social ainda se diferenciam no modo tratar das questões relacionadas ao público.

Nesse aspecto, reflete-se a importância de que haja um fortalecimento da articulação intersetorial entre os serviços com alinhamento do cuidado desenvolvido, por meio do fortalecimento da comunicação, reuniões sistemáticas, encaminhamento com informações mais detalhadas a respeito de cada usuário, potencializar os espaços que já existem nos dois serviços, além de apoio matricial para os casos mais complexos.

Ao longo das oficinas surgiram diversas dificuldades, tais como disponibilidade de horários, espaço adequado e alguns posicionamentos proibicionistas por parte de alguns profissionais. Nessa lógica, observa-se a limitação de se desenvolver atividades práticas e teóricas no ambiente de trabalho, sendo fundamental que a micro gestão se envolva e participe do que está sendo discutido a respeito dos processos de trabalho. Somado a isso, têm-se a ideia da complexidade atrelada à temática.

Neste contexto, mesmo diante dos obstáculos, compreende-se que a utilização da problematização, com o apoio do Arco de Maguerez, mostrou-se muito valiosa no desenvolvimento das oficinas, sendo apontada como um método simples, mas que gerou importantes reflexões acerca do processo de trabalho.

No entanto, identificou-se a necessidade de mais momentos e pesquisas realizadas diante da complexidade da temática e da necessidade de fortalecimento da PRD no âmbito do cuidado desse público. Dentre elas, a importância de se envolver os profissionais da APS nas discussões.

\section{Referências}

1. Tondin MC, Neta MAPB, Passos LA. Street Cabinet: away of intervention to drug use with homeless people. Rev Educ Pública. [Internet]. 2013 May-Aug; 22(49/2): 485-501. [cited Nov 15 2018]. Avaliable from: http://periodicoscientificos.ufmt.br/ojs/index.php/ educacaopublica/article/view/929.

2. Ferreira CPS, Rozendo CA, Melo GB. A Street Clinic in a state capital in Northeast Brazil from the perspective of homeless people. Cad Saúde Pública. 2016 Aug; 32(8): 2595-06. doi: http://dx.doi. org/10.1590/0102-311X00070515.

3. Santos FF, Ferla AA. Mental health and primary care in alcohol and drug users care. Interface. (Botucatu). 
2017 Feb; 21(63): 833-44. doi: http://dx.doi. org/10.1590/1807-57622016.0270.

4. Souza SEF, Mesquita CFB, Sousa FSP. Street approach to people who use psychoactive substances: an experience report. Saúde em Debate. 2017 Jan-Mar; 41(112): 331-9. doi: http://dx.doi. org/10.1590/0103-1104201711226.

5. Aguiar KF, Rocha ML. Participatory action research and the production of new analysis. Psicol Cienc Profissão. 2003 Dec; 23 (4): 64-73. doi: https://doi.org/10.1590/ S1414-98932003000400010.

6. Bordenave JD, Pereira AM. Estratégias de ensinoaprendizagem. 32 ed. Petrópolis (RJ): Vozes; 1986. 302 p. 7. Bessa S, Castro EAS, Gonçalves JR. Methodology of problematization in the course of pedagogy: a report of experience. Rev Profissão Docente. 2017 Aug- Dec; 17(37): 102-14. doi: https://doi.org/10.31496/rpd.v17i37. 8. Ministério da Saúde (BR). Resolução n 466, de 12 de dezembro de 2012. Dispõe sobre pesquisa envolvendo seres humanos. [Internet]. Brasília: Ministério da Saúde; 2012. [Acesso 25 jan 2018]. Disponível em: https:// conselho.saude.gov.br/resolucoes/2012/Reso466.pdf.

9. Wijk LM, Mângia EF. Health care for Homeless People by the Network of Psychosocial Attention of Sé. Saúde em Debate. 2017 Oct-Dec; 41 (115): 1130-42. doi: http://dx.doi.org/10.1590/0103-1104201711511.

10. Salgado RRSP, Fuentes-Rojas M. Homeless population and Mental Health: challenges in the construction of a singular therapeutic plan. Serv Soc Saúde. 2018 Jul-Dec; 17 (2): 250-65. doi: https://doi. org/10.20396/sss.v17i2.8652111.

11. Castro LZ, Andrade MC, Chernicharo RL. A população em situação de rua e a busca pelo sentido da vida: uma questão de sobrevivência. Pretextos- Rev da Graduação Psicol da PUC Minas. [Internet]. 2018 Oct; 3(6). [Acesso 23 jan 2019]. Disponível em: http:// periodicos.pucminas.br/index.php/pretextos/article/ view/16001/13619.

12. Antunes CMC, Rosa AS, Brêtas ACP. From the stigmatizing disease to resignification of living on the streets. Rev Eletrôn Enferm. 2016 Mar; 18: 1-9. doi: https://doi.org/10.5216/ree.v18.33141.

13. Calassa GDB, Penso MA, Freitas MD. Harm reduction in the view of professionals working in CAPS AD II of the Federal District. Psicol Pesqui. 2015 Dec; 9(2): 177-87. doi: http://dx.doi.org/10.5327/ Z1982-1247201500020008.

14. Lopes MA, Sprícigo JS, Mitjavila MR, Schneider DR, Abreu D. Differences in age and gender among CAPS ad users and the implications in the health care network. SMAD, Rev Eletrônica Saúde Mental Álcool Drog. 2018 Jul.-Sep; 14(3): 159-67. doi: https://dx.doi. org/10.11606/issn.1806-6976.smad.2018.000412.
15. Lisboa MS. Os loucos de rua e as redes de saúde mental: os desafios do cuidado no território e a armadilha da institucionalização. [Tese Doutorado em Psicologia Social]. São Paulo (SP): Pontifícia Universidade Católica de São Paulo; 2013. 290 p. [Acesso 23 jan 2018]. Disponível em: https://tede2.pucsp.br/bitstream/ handle/17023/1/Milena\%20Silva\%20Lisboa.pdf.

16. Foucault M. Microfísica do Poder. Cambridge: Harvard UP; 1980.4 ed. Rio de Janeiro (RJ): Edições Graal; 1979. 174 p.

17. Silva LG, Calheiros PRV. Practices employed in treatments of psychoactive substances addicts in Brazilian therapeutic communities: a literature systematic review between 2005-2015. Cad Bras Saúde Mental. [Internet] 2017; 9(23): 67-83. [cited Jan 26 2020] Avaliable from: https://periodicos.ufsc.br/index. php/cbsm/article/view/69370.

18. Thofehrn MB, Montesinos MJL, Arrieira IC, Àvila VC, Vasques TCS, Farias ID. Nurses' work process in a hospital in Spain: emphasis on the technologies of care. Cogitare Enferm. 2014 Jan-Mar; 19(1): 141-6. doi: http://dx.doi.org/10.5380/ce.v19i1.35972.

19. Neves ET, Okido ACC, Buboltz FL, Santos RP dos, Lima RAG de. Accessibility of children with special health needs to the health care network. Rev Bras Enferm. 2019 Dec; 72 (Suppl 3): 71-7. doi: https://doi. org/10.1590/0034-7167-2017-0899.

20. Lemke RA, Silva RAN. Routes for the construction of a territorial logic of care. Psicol Soc. 2013; 25(Supl. 2): 9-20. doi: http://dx.doi.org/10.1590/ S0102-71822013000600003.

21. Ministério da Saúde (BR). Portaria no 121, de 25 de janeiro de 2012. Institui a Unidade de Acolhimento para pessoas com necessidades decorrentes do uso de Crack, Álcool e Outras Drogas (Unidade de Acolhimento), no componente de atenção residencial de caráter transitório da Rede de Atenção Psicossocial [Internet]. Brasília: Ministério da Saúde; 2012. [Acesso 23 Jan 2020]. Disponível em: http://bvsms.saude.gov.br/bvs/ saudelegis/gm/2012/prt0121_25_01_2012.html.

\section{Contribuição dos autores}

Concepção e desenho da pesquisa: Lorenna Saraiva Viana, Eliany Nazaré Oliveira, Maria Suely Alves Costa, Claudine Carneiro Aguiar, Roberta Magda Martins Moreira e Andriny Albuquerque Cunha. Obtenção de dados: Lorenna Saraiva Viana e Eliany Nazaré Oliveira. Análise e interpretação dos dados: Lorenna Saraiva Viana, Eliany Nazaré Oliveira, Maria Suely Alves Costa, Claudine Carneiro Aguiar, Roberta Magda Martins Moreira e Andriny Albuquerque Cunha. Redação do manuscrito: Lorenna Saraiva Viana, Eliany Nazaré Oliveira, Maria Suely Alves Costa, Claudine Carneiro Aguiar, Roberta 
Magda Martins Moreira e Andriny Albuquerque Cunha. Revisão crítica do manuscrito quanto ao conteúdo intelectual importante: Lorenna Saraiva Viana, Eliany Nazaré Oliveira, Maria Suely Alves Costa, Claudine Carneiro Aguiar, Roberta Magda Martins Moreira e Andriny Albuquerque Cunha. Orientação da pesquisa: Eliany Nazaré Oliveira.

Todos os autores aprovaram a versão final do texto.

Conflito de interesse: os autores declararam que não há conflito de interesse. 